\title{
Leer y escribir en la Universidad: una propuesta interdisciplinar
}

\author{
M.Sc. Pablo Arnáez Muga' \\ pam@netuno.net.ve
}

\section{RESUMEN}

La escritura, proceso complejo y valorado socialmente, es entendida grosso modo como una habilidad adquirida y desarrollada en las primeras etapas del sistema educativo $y$, en consecuencia, el nivel superior recibe usuarios capaces de demostrar sus competencias discursivas y textuales. Después de once años de escolàridad, esta pareciera ser la realidad; sin embargo, los hechos demuestran que los escritos universitarios presentan carencias en lo conceptual, lo estructural-textual y lo formal. Ante esta situación, la cultura escrita en la universidad exige una concienciación institucional y personal (alumnos y docentes), razón por la cual se propone reflexionar sobre esta realidad y ofrecer un plan de trabajo interdisciplinario entre la lengua y las otras áreas del conocimiento. Autores como Creme y Lea (2000), Narvaja de Arnoux (2002), Carlino (2005) y Cassany (2006) apoyan teóricamente este trabajo, pues defienden el papel de la lectura y la escritura en el nivel superior y hacen propuestas que ayudan a impulsar una renovación en la valoración y el tratamiento de la lectura y la escritura universitarias. Metodológicamente, un trabajo de campo en el área de Matemática permitió elaborar un proyecto factible. Se aplicaron y valoraron cuatro cuestionarios y, con base en esos resultados, se diseñó la propuesta orientada por la secuencia didáctica. En conclusión, la lectura y la escritura deben ser tratadas de manera interdisciplinar y como compromiso de todos, pues existen necesidades específicas sobre textos académicos especializados para las que debe aplicarse un proyecto de lectura y escritura relacionado con el currículo y las disciplinas.

\section{Palabras clave}

Cultura escrita universitaria, textos académicos, escritura disciplinar.

\section{ABstract}

Writing, a complex and socially valuable process, is understood as an acquired ability developed in the first stages of the educational system. This means that once students get to university, they are already prepared to show their discursive and textual competences. This should be the case after eleven school years; however, what the facts show is that university writing production presents many flaws at conceptual, structural-textual and formal levels. This being the case, written culture in university demands a personal and institutional compromise to build up a conscience towards writing; for this reason, it is necessary to think upon this reality and to offer an interdisciplinary plan in which the area of language works in tandem with other areas of knowledge. The plan proposed in this paper is theoretically supported by authors like Creme and Lea (2000), Narvaja de Arnoux (2002), Carlino (2005) and Cassany (2006), for they defend the roll of reading and writing at university level and make proposals which help to promote a renewal in the value and treatment of university reading and writing. Methodologically speaking, a field-work in the area of mathematics allowed us to elaborate a feasible project. Four questionnaires were valued and applied, and based upon those results a didactic-based proposal was designed. To conclude, reading and writing must be dealt with in an interdisciplinary way and as a compromise assumed by everyone, as there are specific needs about specialized academic texts which may be met by the appliance of a reading and writing project related with the curriculum and the rest of the disciplines.

\section{KEYWORDS}

Written culture in university, academic texts, disciplinary writing.

1 Magíster en Lingüística. Coordinador de la línea de investigación "Lingüística aplicada a la enseñanza de la lengua" en el cillhom. Miembro del Departamento de Lengua y Literatura de la Universidad Pedagógica Experimental Libertador (núcleo de Maracay-Venezuela). Miembro del Centro de Investigaciones Lingüísticas y Literarias Hugo Obregón Muñoz (CILlHoM). Investigación: "La escritura en la Universidad". 


\section{Introducción}

$\mathrm{L}$

a lectura y la escritura son dos procesos

fundamentales en la historia del hombre, en el desarrollo de la humanidad y en la adquisición y estructura de nuevos conocimientos (Olson, 1998). Ahora bien, adentrarnos en la cultura escrita que se imparte, predomina y se cultiva en el ámbito universitario constituye toda una experiencia que cada día se convierte en objeto de interés tanto para los docentes como para los discentes.

\section{Adentrarnos en la cultura escrita que se imparte, predomina y se cultiva en el ámbito universitario constituye toda una experiencia que cada día se convierte en objeto de interés tanto para los docentes como para los discentes.}

En algunos documentos e informes de la UNESCo (1990 y 1995), se destaca el valor y la importancia que para el desarrollo integral del individuo tienen la adquisición, desarrollo y dominio de las habilidades de la lectura y la escritura. Si en el Informe a la unesco de la Comisión Internacional sobre la Educación para el Siglo XxI, presidida por Jacques Delors (1997), intitulado La educación encierra un tesoro, se destacaba el conjunto de conocimientos que debe adquirir el educando centrados en el "aprender a ser, aprender a hacer, aprender a conocer y aprender a convivir", no es menos cierto que el dominio de los procesos productivos y comprensivos entre los universitarios reclama una toma de conciencia y una preparación acorde con las exigencias de los nuevos tiempos.

Detrás de los sistemas de escritura, analizados lingüísticamente por Sampson (1997), hay implicaciones comprensivas y productivas que van más allá del simple deletreo y de la mera trascripción grafemática. Tanto en las áreas productivas (hablar y escribir) como en las comprensivas (escuchar y leer) existen procesos cognitivos y metacognitivos (Flower y Hayes, 1980 y 1981) sobre los que los docentes, en su papel de mediadores (Vygotsky, 1995), y los discentes deben concienciar para alcanzar la comprensión en la lectura y elaborar textos con adecuación, coherencia, cohesión y sin demeritar los aspectos estilísticos y formales.

Frente a la necesidad de que los seres humanos pasasen de la oralidad, como forma de comunicación, a otras formas de codificar los mensajes y entenderlos, surgen los estudios teóricos sobre la lengua escrita (Catach, 1996), las explicaciones en cuanto a cómo se construye la escritura (Ferreiro, 1982) y los modelos cognitivos, explicativos e instructivos de la composición escrita (Cassany, 1990; Bereiter y Scardamalia, 1992; Parodi, 1999; Hernández y Quintero, 2001, y García y Marbán, 2002). Todas estas concepciones, al menos a nivel teórico, han tenido y tienen gran aceptación en los programas de los niveles primarios o básicos y en las escuelas.

También existe toda una corriente que desea rescatar el valor de la escritura en la educación secundaria. Al respecto, baste mencionar los estudios de Björk y Blomstand (2000). El estudio de estos autores, en la primera parte, recurre a la presentación de los modelos metodológicos y a los enfoques didácticos que facilitan el proceso de escribir y, en la segunda, abordan las estructuras textuales más utilizadas por los alumnos de ese nivel académico: desde la narración, pasando por el resumen, hasta abordar los textos literarios y los trabajos de investigación. Sobre este particular hay experiencias en Chile (Peronard, et al., 1998), en Colombia (Pérez Grajales, 1999 y Martínez, 2004), en Argentina (la revista Lectura y $V i d a$ es una buena vitrina para observar experiencias interesantes al respecto) y en Venezuela (Vid Serrón, 1998), por nombrar algunos países.

Y después de once o doce años de escolaridad y, se supone, de orientación, interacción y apropiación de los procesos productivos y comprensivos, esos alumnos de secundaria devienen en estudiantes universitarios. Leer y escribir en la universidad se han convertido en actividades que a diario ejecutan los estudiantes de todas 
las carreras y de todas las áreas del conocimiento. Quizás por considerarlas inherentes al hecho educativo y por ser expresión de una escolaridad (primaria y secundaria), es por lo que se valoran como competencias adquiridas en las que los docentes universitarios poco o nada pueden decir y hacer. Premisa falsa, pues en atención al criterio de especialistas en este tema (Carlino, 2005 y Cassany, 2006), las prácticas discursivas exigidas en la universidad responden a nuevas necesidades y la comprensión y producción de esos nuevos textos exigen nuevas competencias.

Es cierto que, desde la década de los ochenta, los diseños curriculares de los ministerios de educación y los programas de Lengua y Literatura responden al aggiornamiento exigido por los nuevos enfoques y modelos que conciben la lectura y la escritura bajo la perspectiva de lo cognitivo, lo procesal y lo ecológico. Sin embargo, la realidad de los que ingresan al sistema de educación superior es desalentadora y más aún cuando las evaluaciones realizadas a los docentes de aula egresados de las universidades arrojan resultados muy deficientes (Cárdenas, 1995).

Estas realidades presupuestas en lo teórico, en lo metodológico y en los resultados de los egresados de las universidades constituyen el punto de partida para abordar los problemas de la cultura escrita en la universidad y más en concreto en la Universidad Pedagógica Experimental Libertador (UPEL-Maracay). Se acepta como premisa el hecho de que un curso de lengua española (es lo que se ofrece a todas las especialidades en la UPEL-Maracay) no puede subsanar las carencias de muchos años $y$, además, no es el ámbito adecuado para abordar los problemas específicos de las producciones textuales que necesita cada especialidad.

Nuestra hipótesis de trabajo se sostiene sobre dos asertos: (a) la enseñanza de la lectura y la escritura debe hacerse a través del currículo y de manera interdisciplinar y (b) nadie mejor que los especialistas de las distintas áreas del conocimiento que se imparten en las instituciones universitarias para poder interactuar con los especialistas de la lengua. Es por ello que, dentro del marco de esta hipótesis que orienta el devenir de la investigación, nos proponemos, en primera instancia, hacer una reflexión sobre el valor e importancia que algunos especialistas le conceden a la escritura y a la lectura en la universidad; en segundo lugar, diagnosticar en el Departamento de Matemática las necesidades textuales que requiere el desenvolvimiento adecuado de sus integrantes como comprensores y productores de textos $y$, finalmente, proponer el trabajo interdisciplinar como una alternativa para trabajar la lengua en función de las otras unidades curriculares.

\section{Fundamentos teóricos}

\subsection{Una realidad}

Los estudiantes que cursan estudios en las Facultades de Humanidades o en los Departamentos de Lengua y Literatura, por la naturaleza de los pénsums de sus especialidades, deben cursar un conjunto de asignaturas orientadas a su preparación académica y a su desenvolvimiento profesional, a la vez que desarrollan sus competencias textuales comprensivas y productivas. No obstante el aserto anterior, las carencias en el manejo de la lengua afloran también en esta comunidad académica (Carlino, 2005).

Ahora bien, si pensamos en facultades como las de Ingeniería, Medicina, Arquitectura, Derecho y otras, nos encontramos con que los cursos que obligan al contacto formal y sistemático con la lengua, sus componentes y su uso se reducen a una o dos asignaturas básicas que todos deben aprobar. El caso de Lengua Española (curso obligatorio del plan de estudios de la UPEL) puede resultar paradigmático. En su propósito general dice lo siguiente:

El curso de Lengua Española ha sido diseñado para desarrollar las habilidades y destrezas lingüísticas que permitan al estudiante desenvolverse de manera efectiva en cualquier situación comunicativa sea oral o escrita. Así como también adquirir competencias lectoras que le permitan asumir una posición crítica y reflexiva ante cualquier tipo de texto (UPEL, 2004).

Pensar que, en dieciséis semanas y con grupos muy heterogéneos en sus competencias epistemológicas específicas, se pueden subsanar las carencias que nuestros estudiantes traen del 
Ciclo Diversificado y que, al mismo tiempo, se puede alcanzar lo que el propósito establece, suena utópico.

Hablar de enseñar a leer y escribir en la universidad, en nuestro contexto, se valora peyorativamente, pues se considera que esa es una actividad propia de los primeros niveles de la educación inicial y de la educación básica y que nuestra meta es impartir el conocimiento específico de la ciencia a la que estamos adscritos. No resulta extraño oír: "Para aprender a leer y escribir, han tenido once años", “qué han hecho en la educación primaria y secundaria?" Si se entiende la lectura como un proceso cerrado que se alcanza en un determinado momento de la vida, tendrían sentido las afirmaciones anteriores. Pero la realidad es que los estudiantes universitarios, a partir de las competencias lectoras y escritoras que poseen, deben adentrarse en el tratamiento de determinados géneros discursivos que requieren de los usuarios nuevas competencias. Esto es lo que Narvaja de Arnoux (2002) denomina "letrismo académico" y Carlino (2005) "alfabetización académica".

Tanto en lectura como en escritura, las prácticas discursivas que debe afrontar el estudiante universitario ofrecen un amplio y comple-

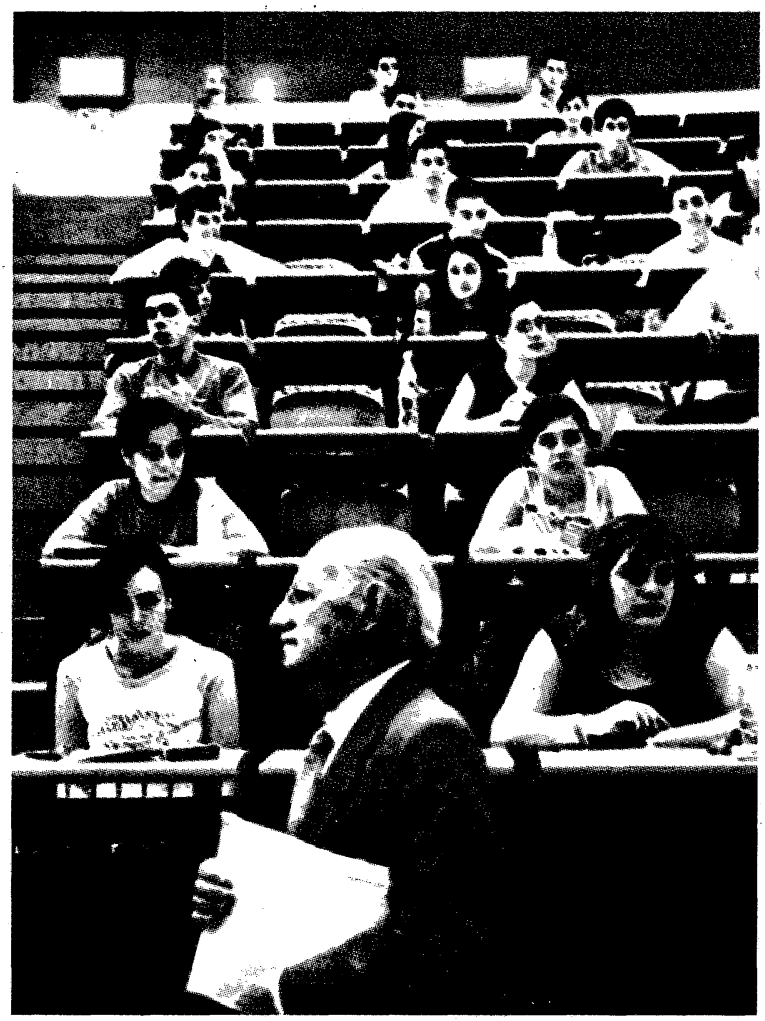

jo abanico de posibilidades y, en consecuencia, el espacio universitario también requiere de una atención especial a la hora de enfrentar los procesos de lectura y escritura, pues las exigencias académicas universitarias son distintas a las requeridas en los niveles y etapas anteriores.

\subsection{Leer y escribir en la universidad}

Autores como Creme y Lea (2000), Narvaja de Arnoux et al. (2002), Carlino (2005) y Cassany (2006) plantean la necesidad de romper el mito de que, una vez adquiridos los principios básicos de.la lectura y de la escritura, se pueden aplicar en todas las situaciones y contextos en los que deba actuar el usuario de su lengua.

A la desmitificación anterior han contribuido la lingüística textual y el análisis del discurso (Bajtin, 1982; van Dijk, 2000 y Martínez, 2004 y 2005). En ambos campos de los estudios lingüísticos, todos los autores coinciden que en cada tipo de texto implica unas condiciones textuales y contextuales que los diferencian a la hora de elaborarlos. Cada producción escrita viene condicionada por el tema, el interlocutor, la superestructura, el propósito y los modos de elaboración (Hymes, 1979 y Casalmiglia y Tusón, 1999). Y cada producción escrita, aun tratándose del mismo tipo de texto, por ejemplo un ensayo, varía en los distintos campos del saber, pues la literatura, la biología nuclear, la física, la filosofía o la historia de la humanidad tienen requerimientos y particularidades que de alguna manera el escritor debe tomar en consideración.

Las autoras Creme y Lea (2000) nos presentan su libro de una manera muy pedagógica y fusionando las especificaciones teóricas con las actividades y ejemplos prácticos. A su vez, además de valorar las prácticas de redacción que se hacen en las aulas desde la escuela primaria, hacen hincapié en que redactar en la universidad exige un nuevo lenguaje, otros metalenguajes y unas formas más diferenciadas de abordar el texto en consonancia con las necesidades académicas de su área de conocimiento.

A su vez, Narvaja de Arnoux et al. (2002) trabajan con los distintos géneros discursivos de los que los estudiantes deben apropiarse a lo largo de su vida académica universitaria. La mo- 
dalidad de taller es la que les sirve a las autoras para ejercitar la lectura y la escritura mediante pautas y registros que ayudan a desarrollar, por un lado, los procesos cognitivos y metàcognitivos involucrados en la lectura interpretativa y, por el otro, las capacidades inherentes a la producción de textos propios de su esfera social.

Por su parte, Carlino (2005) defiende la tesis de que la lectura y la escritura no pueden ser una responsabilidad achacada únicamente a alguno de los compromisarios en el proceso: alumnos, docentes e institución. La responsabilidad debe ser compartida y asumida por todos en una conjunción de acciones. La autora establece la relación existente entre leer, escribir y aprender, términos que le dan título a su libro Escribir, leer y aprender en la universidad. Una introducción a la alfabetización académica, y sugiere que los alumnos adquieran protagonismo en la acción cognitiva y los docentes enfaticen en "los procesos y prácticas discursivas y de pensamiento" (13). Se trata de que docentes y estudiantes se apropien de la "alfabetización académica" comó un modo de hacer con la lectura y la escritura en el ámbito académico universitario y fuera de él.

Un autor que, desde finales de la década de los ochenta, viene trabajando con la escritura en sus aspectos teóricos, metodológicos y didácticos es Cassany. En el año 2006, este autor nos propuso, también a través de la técnica del taller y del comentario de texto, el trabajo con los distintos géneros discursivos en atención a las exigencias de cada una de las disciplinas. Como el mismo autor lo manifiesta: "En cualquier disciplina usamos textos para aprender su conocimiento; sus prácticas laborales requieren aprender a saber interpretar y producir sus escritos" (12).

Los autores brevemente reseñados defienden el papel de la lectura y la escritura en el nivel superior y hacen propuestas que ayudan a impulsar una renovación en la valoración y el tratamiento de la lectura y la escritura universitarias.

En esta misma línea de acción de los autores mencionados, conviene crear conciencia de la cultura escrita en la universidad, para lo cual se propone el compromiso institucional y la acción interdisciplinar entre los actores competentes en el manejo de la lengua. (especialmente en el uso) y los docentes y alumnos de las distintas especialidades.

La lectura y la escritura tienen razón de ser en contextos específicos y en situaciones concretas en las que los individuos, en este caso estudiantes universitarios de diversas facultades o especialidades, interactúan con textos especializados de su área de conocimiento. Esos textos deben ser comprendidos para apropiarse de los contenidos y de los nuevos saberes que proporciona la lectura, los cuales deben convertirse en insumos para producir unos nuevos textos exigidos por los docentes, por las cátedras $\mathrm{y}$, en definitiva, por la comunidad académica a la que pertenecen. En suma, la lectura y la escritura deben ser herramientas fundamentales en el estudio de los temas curriculares y para ello se propone el trabajo interdisciplinar.

\section{Método y procedimientos}

La propuesta que se presenta pretende conjugar las necesidades de una determinada área del conocimiento (en este caso de Matemática) con la comprensión y producción de un determinado texto: La resolución de problemas.

Desde el punto de vista metodológico, se trabajó con el enfoque de un proyecto factible en el que se involucraron el docente de matemática, el docente de lengua y los estudiantes del curso seleccionado. Una vez constatada la factibilidad de la propuesta, de las fases de diagnóstico, diseño, ejecución y evaluación, a los efectos del presente artículo, se hizo énfasis en las dos primeras.

La fase diagnóstica permitió conocer la realidad de los docentes y estudiantes de la Universidad Pedagógica Experimental Libertador, núcleo Maracay (UPEL-Maracay) en cuanto a sus actitudes y aptitudes en el Departamento de $\mathrm{Ma}$ temática con respecto a la lectura y a la escritura. 
Para ello, y con sustento teórico de los trabajos de Cassany (1999), se elaboraron cuatro cuestionarios: (a) Estrategias de composición (Anexo A), (b) Lo que piensan los estudiantes (Ànexo B), (c) Actitudes de los participantes (Anexo C) y (d) Estructuras textuales requeridas (Anexo D). La descripción de los resultados se hizo de manera cualitativa y cuantitativa a partir de la frecuencia y porcentajes de las variables estudiadas. Estos cuestionarios se aplicaron a una sección de Matemática de la especialidad de Educación Integral.

La fase de diseño, en la que se presenta la propuesta, consistió en relacionar la resolución de problemas (el texto surgido de las necesidades de los estudiantes del Departamento de Matemática) con la secuencia didáctica como estrategia de trabajo para interactuar con los estudiantes e involucrarlos en el objetivo de comprender y producir el texto seleccionado.

\section{Propuesta y análisis de los resultados del diagnóstico}

Para llevar a efecto la primera fase de la propuesta se ejecutó el diagnóstico del grupo seleccionado. Se les aplicaron cuatro cuestionarios orientados a detectar: (a) la manera en que cada uno concibe el acto de escribir, (b) el conocimiento sobre la escritura, (c) las actitudes y aptitudes de los estudiantes así como a las estrategias que utilizan a la hora de comprender y producir un texto escrito, y (d) las prácticas discursivas requeridas por este grupo humano.

\section{Cuestionario 1: Estrategias con composición}

Este cuestionario ofrece un conjunto de 10 proposiciones sobre las estrategias de composición que utiliza el participante y que debe seleccionar según sea su comportamiento.

Interesa conocer qué hace el escritor ante factores como los modelos, el lector, los borradores, los planes y esquemas previos a la escritura, las preferencias entre la forma y el contenido, las consultas, los diversos contextos y situaciones de escritura, y el papel de la lectura, entre otros.

$\mathrm{El}$ análisis de las respuestas del cuestionario 1 nos permite afirmar que los alumnos, en su

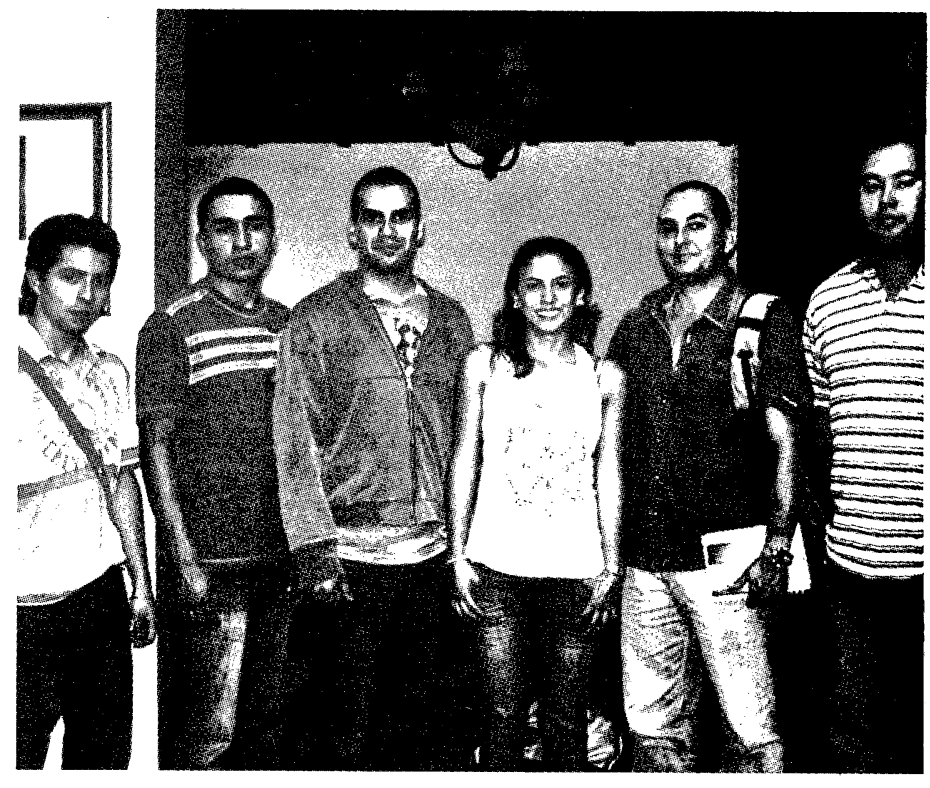

gran mayoría, obvian la lectura de textos modélicos y proceden sin un plan preconcebido y sin tener en cuenta los procesos de planificación, redacción y textualización. Unos pocos (un $23 \%$ ) hacen un esquema previo antes de empezar a escribir y la revisión del escrito se reduce a algunos aspectos de forma cuando escriben a mano y cuando lo hacen con el ordenador lo dejan a merced de la función "ortografía”.

\section{Cuestionario 2: Lo que piensan los estudiantes}

También se elaboró un cuestionario para conocer qué piensan los estudiantes universitarios sobre un conjunto de afirmaciones (unas verdaderas y otras falsas) que se les proporcionan con respecto a la escritura.

El cuestionario consta de 17 afirmaciones y es recomendable, según Cassany (1999), que se contesten todas, aunque no se sepa la respuesta o se tengan dudas, pues es una manera de motivar y propiciar el comentario posterior entre participantes y docente.

En lo referente al cuestionario 2, es interesante constatar que el $44 \%$ de las afirmaciones son contestadas incorrectamente. Dos ejemplos nos mostrarán lo que piensan los estudiantes del acto de escribir. El $90 \%$ de los participantes consideran que el proceso de la escritura es lineal, con lo cual la recursividad, la planificación, los borradores, la relectura y la revisión quedan en un segun- 
do plano. En la práctica, a la mayoría les preocupa más la forma que el contenido de lo que escriben.

\section{Cuestionario 3: Actitudes de los participantes}

El tercer cuestionario pretende analizar y comentar las actitudes sobre la composición escrita que tienen los participantes frente a la escritura. Consta de 20 ítems que de alguna manera intentan reflejar actitudes positivas y negativas con respecto a la composición escrita. Puede ser ilustrativo y de gran utilidad comentar los resultados y reflexionar sobre ellos.

El estudio de las respuestas al cuestionario 3 muestra el bajo nivel que tiene la parte actitudinal de los estudiantes hacia el acto de escribir. En primer lugar, prefieren decir lo que piensan a escribirlo, no les entusiasma escribir y consideran que escribir es algo anticuado e inútil. No hay uniformidad en los resultados.

Para el tema de las actitudes, Cassany (1999: 300) propone la siguiente fórmula: 40 puntos + [suma de las respuestas positivas] - [suma de respuestas negativas] = grado de actitud.

La aplicación de la fórmula y el procesamiento de los datos arrojaron los siguientes resultados que aparecen reflejados en el cuadro 1.

Existe un predominio de la actitud negativa con un $62 \%$. Estos porcentajes deben hacer reflexionar a los responsables de la educación en todos los niveles. También deben cuestionar el trabajo que se hace con la escritura en nuestras aulas. Y, finalmente, deben comprometer a docentes, estudiantes e institución en un trabajo mancomunado para mejorar la parte actitudinal que, a su vez, redunde en lo conceptual y lo procedimental.

\section{Cuestionario 4: Estructuras textuales requeridas}

Se cierra esta primera fase con un cuestionario cuya finalidad es conocer de primera mano, a saber, de los propios departamentos y facultades involucrados, las necesidades textuales que requiere el desenvolvimiento adecuado de sus integrantes como comprensores y productores de textos.

El cuarto cuestionario arrojó como resultado una necesidad muy sentida entre la comunidad de estudiantes y profesores del Departa- mento de Matemática y que no se les propuso entre las alternativas. Se necesitaba abordar "la resolución de problemas", pues, al tiempo que se trata de una estrategia cognitiva y metacognitiva, viene configurada en una forma textual con una serie de elementos verbales, estructurales y semánticos muy específicos.

\section{Propuesta: La resolución de problemas y la secuencia didáctica}

Frente a la realidad de nuestro contexto universitario y frente a las carencias lingüísticas y discursivas que reconocemos en nuestros educandos, se optó por abordar la lectura y la escritura en el ámbito universitario mediante un trabajo interdisciplinario.

Para la resolución de problemas (RP), hemos considerado pertinente tomar como referencia las ideas y propuestas de Pozo y Postigo (1994) y correlacionarlas con la secuencia didáctica (SD). Para los dos autores mencionados, las estrategias de solución de problemas son "concebidas como secuencias de acciones realizadas de modo consciente y deliberado, producto de una reflexión previa” (182). En su consecución, se requiere activar una serie de procesos cognitivos, tales como: estrategias de resolución de problemas, conocimientos conceptuales específicos, metaconocimiento, técnicas y destrezas, algoritmos, estrategias de apoyo, etc.

Por su parte, la SD también exige que una serie de actividades y ejercicios se dispongan de manera gradual para resolver progresivamente las dificultades de los alumnos y para que tomen conciencia de las características del problema que se desea resolver (lingüístico, matemático, químico, biológico, etc.).

La SD y la RP suponen una serie de fases o etapas que conviene seguir para alcanzar la meta propuesta. En el caso de la SD seguimos la propuesta de Camps et al. (2003) y para la RP, la de Polya (citado en Pozo y Postigo, 1994: 188). Veamos el cuadro 2:

$\mathrm{El}$ cuadro precedente nos muestra que la fase $1(\mathrm{SD})$ corresponde a las fases 1 y 2 (RP); la fase $2(\mathrm{SD})$ equivale a la fase $3(\mathrm{RP})$ y la fase $3(\mathrm{SD})$ coincide con la fase 4 (RP). 


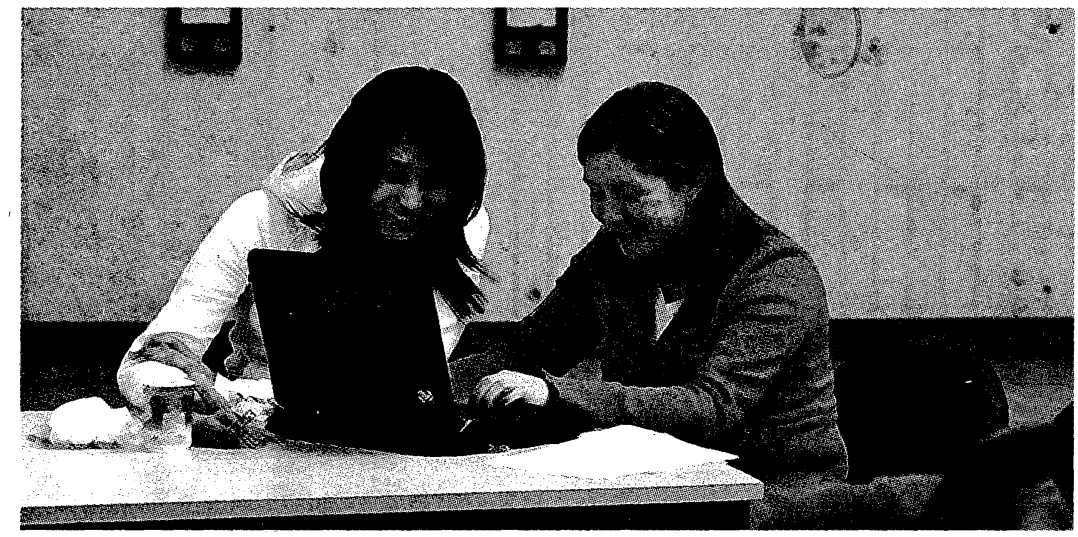

En el caso específico que nos atañe, se propuso la aplicación de la sD tal como se describe en el cuadro 3.

Por tratarse de una SD y de un trabajo interdisciplinar, hay que conjugar el aprendizaje y la enseñanza, lo cual implica: (a) una enseñanza sistemática que facilite los instrumentos comunicativos y lingüísticos; (b) una organización de conjunto (alumnos y docente); (c) una enseñanza intensiva, en términos de Pasquier y Dolz (1996); (d) la negociación de la tarea, los objetivos, los procedimientos y la evaluación, y (e) que el alumno sepa, desde el principio, por qué y para qué está trabajando.

\section{Conclusiones}

Hay que romper la falsa creencia, todavía vigente en los inicios del siglo XXI, de que la lectura y la escritura son conocimientos que corresponden al área de lengua y literatura y que su enseñanza compete única y exclusivamente a los docentes de dicha área.

La lectura y la escritura deben ser tratadas de manera interdisciplinar y como compromiso de todos en la comprensión y producción textual. Los docentes de los conocimientos específicos de un área determinada también se tienen que involucrar, pues son ellos los que conocen la forma de abordar el saber de su especialidad y, además, entienden las prácticas discusivas que más se utilizan en su contexto académico.

Ambas destrezas deben contribuir con el aprendizaje y deben constituirse en herramientas epistemológicas en la adquisición, comprensión y manifestación de los conocimientos.
En el contexto universitario existen necesidades específicas sobre textos académicos especializados y debe aplicarse un proyecto de lectura y escritura relacionado con el currículo y las disciplinas que se imparten en las distintas especialidades y facultades.

El procesamiento de la información configura el deber ser del trabajo universitario. La comprensión textual, la aprehensión de conocimiento científico o humanístico y la producción de discursos coherentes son las metas que los alumnos y docentes deben perseguir.

Se debe instaurar una reflexión a nivel de la comunidad universitaria sobre las prácticas académicas relacionadas con la lectura y la escritura. Este análisis involucra factores de índole institucional, social y político, razón por la cual se deben involucrar y comprometer la universidad como institución, los docentes, los alumnos y los especialistas.

Desde el punto de vista práctico, la secuencia didáctica se puede convertir en una herramienta que propicie la reflexión, la interacción y el compromiso de todos los involucrados. Los resultados de las fases de ejecución y de evaluación, que están en desarrollo, permitirán comprobar, posteriormente, el alcance, la validez y la eficacia de la propuesta. Ó

\section{Bibliografía}

Bajtin, M. (1982), Estética de la creación verbal, México, Siglo XxI.

Bereiter, C. y Scardamalia, M. (1992), "Dos modelos explicativos de los procesos de composición escrita", en Infancia y aprendizaje, núm. 58, pp. 43-64.

Björk, L. y Blomstand, I. (2000), La escritura en la enseñanza secundaria. Los procesos del pensar y del escribir, Barcelona, Graó.

Cárdenas, L. (1995, abril), "La educación que necesitamos", en Investigación y postgrado, vol. 10, núm. 1, pp. 13-20.

Carlino, P. (2005), Escribir, leer y aprender en la universidad. Una introducción a la alfabetización académica, Buenos Aires, Fondo de Cultura Económica. 
Calsamiglia, H. y Tusón, A. (1999), Las cosas del decir. Manual de análisis del discurso, Barcelona, Ariel.

Cassany, D. (1990). "Enfoques didácticos para la enseñanza de la expresión escrita", en Comunicación, lenguaje y educación, núm. $6, \mathrm{pp}$. 63-80.

- (1999), Construir la escritura, Barcelona, Paidós.

- (2006), Taller de textos. Leer, escribir y comentar en el aula, Barcelona, Paidós.

Catach, N. (comp.), (1996), Hacia una teoría de la lengua escrita, Barcelona, Gedisa.

Creme, P. y Lea, M. (2000), Escribir en la universidad, Barcelona, Gedisa.

Delors, J. (1997), La educación encierra un tesoro [Presentación del Informe de la unesco], en: Aprender para el futuro. Desafíos y.oportunidades, Madrid, Santillana, pp. 47-52.

Ferreiro, E. (1982), "Lós procesos constructivos de apropiación de la escritura", en Ferreiro, E. y Gómez Palacio, M. (edits.), Nuevas perspectivas sobre los procesos de lectura y escritura, México, Siglo xxi.

Flower, L. y Hayes, J. R. (1980), "Identifying the Organization of Writing Precesses", en Gregg, L. W. y Steinberg, E. R. (edits.), Cognitive Processes in Writing, Nueva Jersey, Lawrence Erlbaum Associates.

- (1981), "A cognitive Process. Theory of Writing", en College Composition and Communication, vol. 4, núm. 32, pp. 51-72.

García Sánchez, J. y Marbán, J. (2002), Instrucción estratégica en la composición escrita, Barcelona, Ariel Educación.

Hernández, A. y Quintero, A. (2001), Comprensión y composición escrita, Madrid, Síntesis.

Hymes, D. (1979), "On Communicative Competence", en Lee, V. (edit.), Language Development, Londres, Croom Helm/The Open University, pp. 36-62.

Martínez, M. C. (2004), Estrategias de lectura y escritura de textos. Perspectivas teóricas y talleres, Cali, Universidad del Valle.

- (2005), La construcción del proceso argumentativo en el discurso. Perspectivas teóricas y trabajos prácticos, Cali, Universidad del Valle.
Narvaja de.Arnoux, E.; Di Stefano, M. y Pereira, C. (2002), La lectura y la escritura en la universidad, Buenos Aires, Eudeba.

Olson, D. R. (1998), El mundo sobre el papel, Barcelona, Gedisa.

Parodi, G. (1999), "Conexiones entre comprensión y producción de textos escritos: una aproximación psicolingüística", en Parodi, G. (edit.), Discurso, cognición y escritura, Valparaíso (Chile), Ediciones Universitarias de Valparaíso, pp. 59-90.

Pasquier, A. y Dolz, J. (1996), "Un decálogo para enseñar a escribir", en Cultura y Educación, núm. 2, pp. 31-41.

Pérez Grajales, H. (1999), Nuevas tendencias de la composición escrita, Bogotá, Aula Abierta.

Peronard, M. et al. (1998), Comprensión de textos. escritos: de la teoría a la sala de clases, Santiago de Chile, Andrés Bello.

Pozo, J. I. y Postigo, Y. (1994), "La solución de problemas como contenido procedimental en la educación obligatoria”, en Pozo, J. I.et al., La solución de problemas, Madrid, Santillana, cap. 5.

Sampson, G. (1997), Sistemas de escritura. Análisis lingüistico, Barcelona, Gedisa.

Serrón, S. (comp.), (1998), De la cartilla a la construcción del significado, Caracas, Red Latinoamericana de Alfabetización.

unEsco (1990), Conferencia de Jomtien (Tailandia), unEsco.

unesco (1995), Conferencia de Beijing (China), UNESCO.

Universidad Pedagógica Experimental Libertador (2004), Programa de curso. Lengua Española, Maracay.

van Dijk, T. (comp.), (2000), El discurso como estructura y proceso, Barcelona, Gedisa.

Vygotsky, L. (1995), Pensamiento y Lenguaje, Barcelona, Paidós.

\section{Anexos}

\section{Cueștionario 1: Estrategias de composición}

1. Señala una afirmación en cada una de las siguientes preguntas, según como te comportes. 
2. Anota las matizaciones que harías en cada afirmación elegida, en el caso de que no corresponda exactamente a tu forma de trabajar.

3. Con el test acabado, contabiliza tu puntuación final según el baremo.

1) Buscar modelos:

a) Antes de empezar a redactar, siempre busco y leo textos parecidos al que tendré que escribir.

b) Algunas veces busco modelos, cuando tengo que escribir algún texto que no conozco.

c) Nunca busco ni leo textos que pueda usar de modelos.

2) Pensar en mis lectores:

a) Pienso en los lectores de mi texto durante todo el proceso de redacción.

b) Sólo pienso en los lectores antes de comenzar a redactar.

c) Pienso más en las ideas que quiero comunicar que en mis lectores.

3) Buscar ideas al principio:

a) Apunto las ideas tal como se me ocurren.

b) Apunto las ideas primero para mí, con mis palabras, y después busco la mejor forma de explicarlas a los lectores, con sus palabras.

c) Apunto las ideas directamente con las palabras y las expresiones que puedan entender los lectores.

4) Hacer borradores:

a) No hago borradores; escribo directamente en la hoja final y corrijo en ella con corrector líquido.

b) .Hago un solo borrador del texto, que corrijo antes de pasarlo a limpio.

c) Hago varios borradores que corrijo varias veces, según la dificultad del texto.

5) Hacer planes y esquemas y modificarlos:

a) Nunca hago esquemas o planes del texto; me basta con una idea mental para empezar a redactar.

b) Escribo un esquema inicial que algunas veces cambio cuando redacto.

c) A menudo hago varios esquemas y planes del texto y acostumbro a modificarlos mientras escribo, porque se me ocurren ideas nuevas.
6) Leer mientras se escribe:

a) Siempre hago muchas pausas mientras escribo, para leer varias veces cada fragmento ya escrito.

b) Hago algunas pausas para leer una o dos veces sólo algunos fragmentos.

c) No hago pausas mientras escribo y pocas veces leo lo escrito antes de terminarlo.

7) Revisar la forma y el contenido:

a) Reviso sobre todo la forma en palabras y frases: estilo, gramática, ortografía, puntuación.

b) Reviso sobre todo el contenido en oraciones, párrafos y fragmentos extensos: estructura, ideas, sentido global.

c) Reviso tanto la forma como el contenido.

8) Escribir una idea:

a) Frecuentemente reescribo una idea de maneras diferentes, cambiando palabras, puntos de vista, tono, etc.

b) Algunas veces reescribo una oración, cuando no me gusta la primera versión.

c) Casi nunca modifico la primera versión de una idea y, si lo hago, es para corregir sólo alguna falta.

9) Escribir en circunstancias diferentes:

a) Siempre sigo los mismos pasos en el proceso de composición: generar y ordenar ideas, redactarlas y revisarlas.

b) No siempre sigo todos los pasos, pero mantengo siempre un mismo proceso de trabajo.

c) Adapto mi forma de redactar a las circunstancias de cada momento.

10) Consultar manuales:

a) Nunca consulto diccionarios, libros de gramática o manuales, tampoco utilizo verificadores informáticos.

b) Consulto varias veces estos manuales y siempre compruebo la corrección con verificadores informáticos.

c) Sólo en escritos difíciles utilizo manuales o verificadores.

Tomado de: Cassany, D. (1999), Construir la escritura, Barcelona, Paidós, pp. 280-282. 


\section{Cuestionario 2: Afirmaciones sobre el escribir}

\begin{tabular}{|c|c|c|c|}
\hline & Afirmaciones & $\mathrm{V}$ & F \\
\hline 1. & Escribir es la habilidad lingüistica más difícil de aprender. & & \\
\hline 2. & Escribir es la habilidad lingüística que tiene una frecuencia de uso más baja. & & \\
\hline 3. & E1 lenguaje escrito es muy diferente del oral. & & \\
\hline 4. & Las funciones del lenguaje escrito están localizadas en el hemisferio cerebral izquierdo. & & \\
\hline 5. & $\begin{array}{l}\text { A partir de los años setenta se desarrolló en EE.UU. una prolífica investigación sobre el proceso de } \\
\text { composición. }\end{array}$ & & \\
\hline 6. & Estas investigaciones usan métodos experimentales y científicos. & & \\
\hline 7 & Escribir es buscar palabras para expresar las ideas que ya se tienen en mente. & & \\
\hline 8. & La composición es lineal y se divide en: pre-escribir, escribir y re-escribir. & & \\
\hline 9. & Las reglas de normativa tienen poca utilidad durante la composición. & & \\
\hline 10. & Todos los buenos lectores suelen ser buenos escritores. & & \\
\hline 11. & Los escritores expertos se fijan más en el contenido que en la forma al escribir. & & \\
\hline 12. & Los escritores expertos releen más veces lo que escriben mientras escriben. & & \\
\hline 13. & Los escritores expertos suelen utilizar unas mismas técnicas de redacción. & & \\
\hline 14. & $\begin{array}{l}\text { Los escritores expertos tienen más conciencia del futuro lector del texto que escriben que los } \\
\text { aprendices o inexpertos. }\end{array}$ & & \\
\hline 15. & Los escritores inexpertos revisan más sus textos que los expertos. & & \\
\hline 16 & Los escritores inexpertos aprenden mientras escriben. & & \\
\hline 17. & Escribir es preocuparse sobre todo de expresar ideas en un escrito. & & \\
\hline
\end{tabular}

Tomado de: Cassany, D. (1999), Construir la escritura, Barcelona, Paidós, p. 286.

\section{Cuestionario 3: Actitudes sobre la composición escrita}

\begin{tabular}{|c|c|c|c|c|c|c|}
\hline \multicolumn{2}{|r|}{ Afirmaciones } & 1 & 2 & 3 & 4 & 5 \\
\hline 1. & Me hace ilusión tomar nota de mis ideas. & & & & & \\
\hline 2. & No tengo miedo a que se me corrija lo que escribo. & & & & & \\
\hline 3. & Detesto escribir. & & & & & \\
\hline 4. & Si he de expresar una cosa, prefiero escribirla a decirla. & & & & & \\
\hline 5. & Tengo temor a escribir cuando sé que se me corregirá lo que escribo. & & & & & \\
\hline 6. & Me quedo en blanco al empezar a escribir. & & & & & \\
\hline 7. & Expresar mis ideas por escrito me parece una pérdida de tiempo. & & & & & \\
\hline 8. & No me gusta que se evalúen mis escritos. & & & & & \\
\hline 9. & La escritura sólo es otra forma de comunicación. & & & & & \\
\hline 10. & Tengo confianza en mi capacidad de expresar ideas por escrito. & & & & & \\
\hline 11. & Escribir es una forma anticuada e inútil de comunicarse. & & & & & \\
\hline 12. & En la escuela, escribir es una experiencia agradable. & & & & & \\
\hline 13. & Me parece que soy capaz de anotar mis ideas con claridad. & & & & & \\
\hline 14. & Escribir es una habilidad beneficiosa. & & & & & \\
\hline 15. & Comentar mis escritos con otros es una actividad agradable. & & & & & \\
\hline
\end{tabular}




\begin{tabular}{|l|l|l|l|l|l|}
\hline 16. & Paso momentos horrorosos cuando debo ordenar mis ideas en una redacción. & & & & \\
\hline 17. & Cuando tengo algo que decir, prefiero decirlo a escribirlo. & & & & \\
\hline 18. & Escribir será útil en mi futura profesión. & & & & \\
\hline 19. & Me entusiasma escribir. & & & \\
\hline 20. & No soy nada bueno escribiendo. & & & & \\
\hline
\end{tabular}

Leyenda:

1. Estoy muy de acuerdo.

2. Estoy de acuerdo.

3. No lo tengo claro.

4. No estoy de acuerdo.

5. No estoy nada de acuerdo.

Tomado de: Cassany, D, (1999), Construir la escritura, Barcelona, Paidós: 299.

\section{Cuestionario 4: Estructuras textuales requeridas}

\begin{tabular}{|c|c|c|c|c|c|c|}
\hline & & 1 & 2 & 3 & 4 & 5 \\
\hline 1. & Síntesis & & & & & \\
\hline 2. & Ensayos & & & & & \\
\hline 3. & Monografías & & & & & \\
\hline 4. & Reseñas & & & & & \\
\hline 5. & Pruebas (exámenes) & & & & & \\
\hline 6. & Ponencias & & & & & \\
\hline 7. & Informes & & & & & \\
\hline 8. & Proyectos & & & & & \\
\hline 9. & Otro: & & & & & \\
\hline 10. & 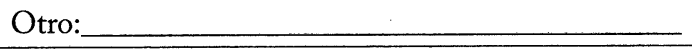 & & & & & \\
\hline
\end{tabular}

\section{Cuadro 1: Actitudes y porcentajes}

\begin{tabular}{|c|c|c|}
\hline Rango & $\%$ & Actitud \\
\hline $00-10$ & - & Muy positiva \\
\hline $10-20$ & 9 & Bastante positiva \\
\hline $20-30$ & 17 & Positiva \\
\hline $30-40$ & - & Regular \\
\hline $40-50$ & 12 & Regular \\
\hline $50-60$ & 41 & Negativa \\
\hline $60-70$ & 20 & Bastante negativa \\
\hline $70-80$ & 1 & Muy negativa \\
\hline
\end{tabular}

\section{Cuadro 2: Fases de la SD y de la RP}

\begin{tabular}{|c|c|c|l|}
\hline \multicolumn{2}{|c|}{ Fases de la sD } & \multicolumn{2}{c|}{ Fases de la RP } \\
\hline \multirow{2}{*}{1.} & Preparación & 1 & Comprender el problema \\
\cline { 2 - 4 } & & 2 & Concebir un plan \\
\hline 2. & Realización & 3 & Ejecutar el plan \\
\hline 3. & Evaluación & 4 & Examinar la solución \\
\hline
\end{tabular}




\section{Cuadro 3: Fases y Subfases de la SD}

\begin{tabular}{|c|c|c|}
\hline Preparación & Realización & Evaluación \\
\hline $\begin{array}{l}\text { 1. Formular el proyecto o tarea a } \\
\text { realizar: } \\
\text { 1.1. el problema que se desea abordar, } \\
\text { 1.2. la situación problemática, } \\
\text { 1.3. el propósito del trabajo, } \\
\text { 1.4. los objetivos del aprendizaje, } \\
\text { 1.5. la forma de lograrlo, } \\
\text { 1.6. evaluación del proceso, del } \\
\text { aprendizaje y del problema resuelto. }\end{array}$ & $\begin{array}{l}\text { Fàses: } \\
\text { 1. Planificación: } \\
\text { 1.1. lectura de problemas, } \\
\text { 1.2. análisis de modelos, } \\
\text { 1.3. contraste, búsqueda y } \\
\text { organización de la información, } \\
\text { 1.4. trabajo grupal, individual o mixto. } \\
\text { 2. Ejecución: } \\
\text { 2.1. discutir la formas de resolución } \\
\text { (alternativas), } \\
\text { 2.2. resolver el problema. } \\
\text { 3. Revisión: } \\
\text { 3.1. contenido, estructura, función y } \\
\text { forma. } \\
\text { 4. Solución definitiva }\end{array}$ & $\begin{array}{l}\text { 1. Evaluar: } \\
\text { 1.1. el proceso de la } \\
\text { resolución del problema, } \\
\text { 1.2. la interacción en el grupo, } \\
\text { 1.3. la mediación del docente, } \\
\text { 1.4. el problema resuelto, } \\
\text { 1.5. lo aprendido: } \\
\text { - en lo conceptual } \\
\text { - en lo procedimental } \\
\text { - en lo actitudinal. }\end{array}$ \\
\hline
\end{tabular}

